\title{
BMJ Open Qualitative study of perspectives concerning recent rehospitalisations among a high-risk cohort of veteran patients in Connecticut, USA
}

Sheila M Antony, ${ }^{1}$ Lauretta E Grau, ${ }^{1,2}$ Rebecca S Brienza ${ }^{1,3}$

To cite: Antony SM, Grau LE, Brienza RS. Qualitative study of perspectives concerning recent rehospitalisations among a highrisk cohort of veteran patients in Connecticut, USA. BMJ Open 2018;8:e018200. doi:10.1136/ bmjopen-2017-018200

- Prepublication history for this paper is available online. To view these files, please visit the journal online (http://dx.doi. org/10.1136/bmjopen-2017018200).

Received 13 June 2017 Revised 27 April 2018 Accepted 4 May 2018

\section{Check for updates}

${ }^{1}$ VA Connecticut Healthcare System, West Haven, Connecticut, USA ${ }^{2}$ Yale School of Public Health, New Haven, Connecticut, USA

${ }^{3}$ Section of General Internal

Medicine, Yale School of

Medicine, New Haven,

Connecticut, USA

Correspondence to

Dr Lauretta E Grau;

lauretta.grau@yale.edu

\section{ABSTRACT}

Objectives Veterans Affairs (VA) patients are at risk for rehospitalisation due to their lower socioeconomic status, older age, poor social support or multiple comorbidities. The study explored inpatients' perceptions about factors contributing to their rehospitalisation and their recommendations to reduce this risk.

Design Thematic qualitative data analysis of interviews with 18 VA inpatients.

Setting VA Connecticut Healthcare System, West Haven Hospital medical inpatient units.

Participants All were aged 18+ years, rehospitalised within 30 days of most recent discharge, medically stable and competent to provide consent.

Measurements Interviews assessed inpatients' health status after last discharge, reason for rehospitalisation, access to and support from primary care providers (PCP), medication management, home support systems and history of substance use or mental health disorders.

Results The mean age was 71.6 years (11.1 SD); all were Caucasian, living on limited budgets, and many had serious medical conditions or histories of mental health disorders. Participants considered structural barriers to accessing PCP and limited PCP involvement in medical decision-making as contributing to their rehospitalisation, although most believed that rehospitalisation had been inevitable. Peridischarge themes included beliefs about premature discharge, inadequate understanding of postdischarge plans and insufficiently coordinated postdischarge services. Most highly valued their VA healthcare but recommended increasing PCPs' involvement and reducing structural barriers to accessing primary and specialty care.

Conclusions Increased PCP involvement in medical decision-making about rehospitalisation, expanded clinic hours, reduced travel distances, improved communications to patients and their families about predischarge and postdischarge plans and proactive postdischarge outreach to high-risk patients may reduce rehospitalisation risk.

\section{INTRODUCTION}

The issue of hospital readmission has come to national attention, and although the link between readmissions and quality of care is controversial, readmissions lead to increased cost, and interventions to reduce

\section{Strengths and limitations of this study}

- Collecting data on Veterans Affairs (VA) patients perspectives about their recent rehospitalisation can identify important contextual factors that are not typically or easily assessed in quantitative studies and may suggest other issues to target in interventions to reduce rehospitalisation risk.

- Inpatients' recommendations about how to reduce rehospitalisation risk may uncover structural/systems issues not recognised by providers or other hospital staff that may be important to target in interventions to reduce rehospitalisation risk.

- Although saturation was achieved, the non-probabilistic sampling strategy and small sample size limits the potential generalisability and should be verified in a larger, quantitative study.

- The study sample included mostly Caucasian males from one VA hospital in the Northeast, and it is possible that other themes exist among non-Caucasian or female patients or in other regions of the USA.

readmissions have been correlated with reduced mortality. ${ }^{1}$ Overall cost for 30-day readmissions within the Veterans Affairs (VA) is estimated at US\$6000 to US\$8000 for an average medical admission, ${ }^{2}$ although some studies suggest higher rates of readmission within the Department of Veterans Affairs health system than in non-VA hospitals. ${ }^{3}$ In addition to overall costs associated with readmissions, rehospitalised patients are more likely to suffer from chronic comorbidities and impaired functional status that place them at increased risk of death. ${ }^{5}$ Systems level factors such as hospital size have been found to be negatively associated with the patient outcomes of rehospitalisation and death. $^{7}$

VA patients may be at higher risk for rehospitalisation due to their lower socioeconomic status, older age, poor social support and multiple comorbidities. ${ }^{8-11}$ Among seriously ill veterans receiving palliative care, a recent 
qualitative study found that issues with self-care and poor support systems may contribute to readmissions. ${ }^{12}$ Chronic disease, distance from the VA and age are also associated with increased readmission risk in veterans. ${ }^{89}$ Studies of transitions of care suggest that difficulty navigating the healthcare system, disempowerment to make health decisions and complex psychosocial factors may contribute to readmissions. ${ }^{13}$ Studies of non-VA patients who are readmitted within 30 days of last discharge reveal that patients often have difficulty in understanding their discharge plans, issues with self-care and difficulty resolving these barriers. ${ }^{1415}$ Functional impairment ${ }^{141617}$ and polypharmacy ${ }^{18}$ are associated with preventable postoperative complications (eg, infection, thromboemboli) ${ }^{19}$ and, among bariatric surgical patients, have higher presurgical basal metabolic index scores. ${ }^{20}$

The VA system has sought to reduce readmission risk, primarily via identification of risk factors in quantitative studies-both within ${ }^{321}$ and beyond the VA system ${ }^{214} 22$ and testing interventions to reduce readmissions. ${ }^{10} 23-25$ For example, rehospitalisation rates were reduced by implementing nursing-led interventions to improve delivery of discharge instructions in patients following hip replacement and pharmacist-led interventions to provide postdischarge medication reconciliation. ${ }^{2325}$ And improved contact with primary care correlated with fewer readmissions among older veterans. ${ }^{10}$ Yet rehospitalisation rates remain high.

To date, few studies have examined veterans' perceptions regarding the readmission experience. ${ }^{12}{ }^{13}$ The current study was undertaken in response to VA interest in exploring patients' perceptions about factors that possibly contributed to their recent rehospitalisation and how to potentially reduce the likelihood of readmission. Identification of the unique challenges and perspectives of these patients may inform future healthcare policies and guide development of interventions aimed at further reducing readmission risk, enhancing quality of healthcare and improving transitions of care.

\section{METHODS}

\section{Setting}

The VA Connecticut (VACT) healthcare system comprises six community-based outpatient clinics (CBOCs), an ambulatory care centre and the 216-bed main hospital and ambulatory care clinics at West Haven and serves 57884 patients. ${ }^{26}$ The study was conducted on the inpatient medicine units at the main hospital, which is staffed by Yale internal medicine residents, full-time hospitalists and rotating primary care and subspecialty attending providers from the West Haven VA.

\section{Patient and public involvement}

The study was not a randomised controlled trial. The research question was based on patients' preference not to be readmitted frequently and the VACT priority to potentially reduce readmissions within 30 days of the last hospitalisation. Neither patients nor the public were involved in the study design. The study goal was to understand the reasons for readmission from patients' perspectives and determine whether we could develop interventions to prevent or reduce readmission. Our initial assumption was that patients would have important insights due to their recent readmission, and the openended interviews allowed participants to shape the discussion according to their own priorities. In addition, patients were not involved in the recruitment or conduct of the study due to patient privacy issues, the uniqueness of the study population (ie, inpatient, readmitted) and the specialised skills required for conducting qualitative interviews. We did not ask participants' permission to contact them after the study due to privacy and HIPAA concerns. The study results will be disseminated through the literature. We will also attempt to distribute the results through regular publications for veterans.

\section{Participant eligibility and recruitment}

Purposive sampling was used to recruit VA patients who were (1) 18+ years of age, (2) rehospitalised to internal medicine within 30 days of last discharge, (3) medically stable and (4) mentally competent to provide consent. Potential participants were identified via retrospective chart review and discussion with the patient's ward nurse to confirm eligibility criteria. Participants were then consented and interviewed during their hospitalisation. Interviews were audiotaped and lasted 20-30 min. Written consent was obtained from all participants.

\section{Data collection and analysis}

An interview guide, previously used in a non-VA setting, ${ }^{27}$ was adapted to include VA-specific questions regarding pharmacy services, telephone triage, inpatient and primary care services and probes to contextualise factors surrounding rehospitalisation. Key domains included perceived postdischarge health status, factors believed to be associated with readmission, medication management, access to and support from primary care, home support systems, resources (eg, housing, transportation) and history of substance use or mental health disorders. Basic demographic information (ie, age, ethnicity, sex) was also recorded. The interviews occurred between September 2013 and October 2014 and were conducted by internal medicine residents with training on qualitative interviewing skills.

All interviews were audiotaped, transcribed verbatim and subsequently deidentified. Interviews continued until data saturation was achieved as determined during regularly scheduled research team meetings. Codebook development and data analysis followed an iterative process and were grounded in the text. The coding and analytic team (SA, LG) met weekly throughout the process of codebook development, coding and analysis. A total of 32 codes were created based on the content expressed during the interviews. Both team members held postgraduate degrees in clinical fields and were experienced in 
qualitative research methods; one (SA) had interviewed some participants and was able to bring that experience to the analytic discussion. Both independently coded all transcripts, and any coding discrepancies were resolved by consensus during the weekly meetings. The team's epistemological position was constructionist and used pragmatism as the interpretive framework. Using ATLAS.ti (V.7.1.7) and thematic analysis, ${ }^{28}{ }^{29}$ we identified common patterns across the dataset, grouped them into themes, and sought 'negative' instances where the data did not fit the existing themes. Reports of all quotes subsumed under each code were generated and discussed iteratively to identify themes that transcended individual codes. The analyses were reviewed by the research team iteratively during the entire coding and analytic period of the study.

\section{RESULTS}

Four major themes were identified. The first related to participants' thoughts about what they valued in their healthcare and providers. Two themes concerned factors that may have contributed to their rehospitalisation, the first primarily identifying factors most closely associated with the actual readmission and the second with factors related to difficulties surrounding discharge and postdischarge services. The final theme concerned their recommendations to reduce rehospitalisation risk.

\section{Sample characteristics}

Table 1 describes the study sample. Of the 18 participants, there were 17 men and 1 woman, proportions that reflect the overall VACT patient population. ${ }^{30}$ All were Caucasian; this is also consistent with the overall racial composition of the West Haven VA patient population. Most were elderly (mean age 71.6; SD 11.1 years), reported being financially secure although often living on limited budgets; approximately half lived with family members or spouses. Many had pre-existing chronic or serious medical conditions (eg, diabetes, pulmonary or cardiovascular disease, neurological disorders, cancer) or histories of affective disorders (eg, depression, post-traumatic stress disorder, anxiety); a few had alcohol or substance use disorders.

Participants could be generally classified into four patient types based on their description of events, behaviours and attitudes: (1) loners, (2) 'hardcore',

Table 1 Sample characteristics $(\mathrm{N}=18)$

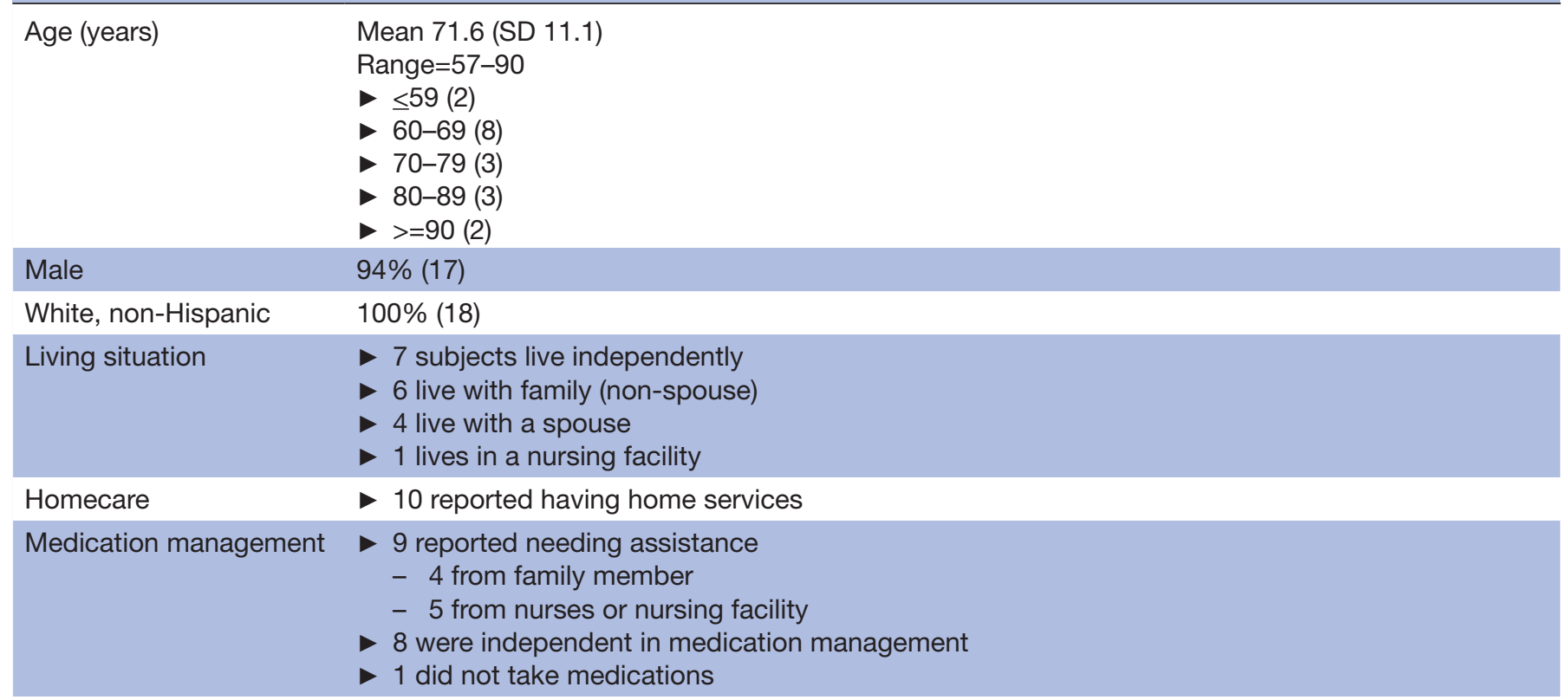

\section{Patient type}

\begin{tabular}{|c|c|}
\hline Loner & $\begin{array}{l}\text { "I deal with my own problems by myself. I've always kept issues to myself". (Male, } 65 \text { years) } \\
\text { "Well, I'm kind of a lonie, you know? I keep to myself". (Male, } 64 \text { years) }\end{array}$ \\
\hline 'Hardcore' & $\begin{array}{l}\text { "I don't go for a hangnail or anything like that. The only time I go over [to the clinic] is when [the } \\
\text { doctor] says, 'It's time for your checkup and they do a little blood work, stuff like that. I'm not the } \\
\text { type of person to go, I'm hardcore". (Male, } 57 \text { years) } \\
\text { "I tough it out and just, there's no reason to call a doctor because you stub your toe". (Male, } \\
64 \text { years) }\end{array}$ \\
\hline Passive/accepting & "I take what they give me. I don’t judge...I figure they know what they're doin'." (Male, 74 years) \\
\hline
\end{tabular}


(3) engaged or (4) passive/accepting. Loners can be described as independently managing their lives (including medical problems) with little or no outside input. 'Hardcore' patients tended to minimise medical problems and delay seeking healthcare services. The engaged patients tended to actively participate in active discussions about their health with their providers. Passive/accepting patients tended to unquestioningly accept medical decisions made by their providers or families. The first three patient types took an active interest in the medical decision-making process, and the fourth and smallest group tended to accept any medical decisions made by their providers or family. Quotes that exemplify each of these patient types are provided in table 1 . The patient types did not neatly 'map' onto the themes, but were thought to influence decision-making processes and activities at times.

\section{Healthcare values and priorities}

Most participants were very satisfied with their healthcare and described their VA providers as '(a) caring person', '(an) excellent team of doctors', '(a) wonderful person' and 'pays attention'. As one male patient aged 69 years noted, 'I think the veterans get a better support system than most [patients] do'.

But [my primary care provider] really does pay a lot of attention. And, to the details. To the minor details that you wouldn't think of. (male, 90 years)

The valued qualities were sometimes explicitly identified or could be inferred from negative statements about their care. Trust was an important quality that participants highly valued, particularly in their primary care provider (PCP).

Because the only one that I want, the only person I want to see when it comes to my health, that are people who I trust. Okay and I know that my primary doctor, I trust, okay. She's very, very good and she's always concerned about me when I walk through that door and she always has a good word to say to me. (Male, 57 years)

And like I told you, I have a lot of faith in [my PCP]. So whatever she says, I go along. (Male, 90 years)

Respectful and attentive attitudes were other commonly valued qualities.

[The PCP is] very good, she'll pick up stuff and she'll call me and check with me and find out what's good and I like that too. (Male, 69 years)

The driver was very disrespectful to me and I says, I don't want you to go and pick on this driver but you get a hold of all your drivers and you sit down with them and they have to respect the veteran who is handicapped. (Male, 57 years)

Participants also valued providers who communicated and listened well and were thoroughly knowledgeable about their case. They appreciated clear and consistent explanations about their medical care, especially in cases involving multiple specialties during their inpatient care.

Well, I always feel better after I talk to my primary care doctor because he knows everything about me. He knows the meds I'm on, he knows about my history. (Male, 64 years)

I mean she was easy to talk to. She, you would tell her your deepest secrets and everything ... that you wouldn't tell nobody else. You just felt comfortable with her, just her personality, her attitude. (Male, 60 years)

[The inpatient medical team will] listen to you for $10 \mathrm{~min}$ and then, okay, I'll get back to you this afternoon and they never do. (Male, 64 years)

Continuity of care was another important issue for participants. For complicated medical problems involving multiple providers or a hospitalisation, some participants questioned the extent of communication between providers and regretted the limited involvement of their PCP during their hospitalisation.

If I'm in a hospital, I think my primary doctor should be one of the first people to come here. (Male, 90 years)

So, it just seems like there's so many people on your case that things get kinda mixed up between the people... I like their 1-on-1, I like the 1-on-1 rather than a team of doctors. (Male, 64 years)

[The] bigger question I have is whether the doctors who prescribe them are talking to each other. (Male, 69 years)

\section{Themes linked to the readmissions event}

Perceptions about the readmission situation included three themes: (1) logistical/structural barriers to accessing their PCP, (2) limited involvement of PCPs in the medical decision-making process and (3) the perceived inevitability of the readmission.

Logistical/structural barriers

Logistical/structural barriers were the most frequently cited problem and mainly concerned challenges to connecting with their PCP in a timely fashion. Participants reported often feeling worse after clinic hours or on weekends when the possibility of speaking to a clinic provider was reduced. Beliefs about limited clinic hours and heavily booked outpatient schedules were other perceived barriers to accessing primary care services.

What happens sometimes, things happen on the weekend...You can't get nobody. (Male, 82 years)

I went over there to see [the PCP] and they told me I couldn't see him. I could only see him by appointment. And they wanted to give me an appointment in, like, a month and a half. (Male, 64 years)

When you get hurt, you get hurt. And if it comes down to it, they're not open, they're only open from 
7:00, 7:30 in the morning till 4:00 in the, then you have to go to the Emergency Room. (Male, 57 years)

For others, the lack of specialty services at the CBOCs necessitated travelling long distances to the central clinic. Perceived transportation difficulties or inefficiencies may have caused patients to attempt handling their medical problems at home. This phenomenon was more likely to occur among loner or 'hardcore' patient types.

I have to depend on somebody to give me a ride and both my brothers that give me a ride down here, they're both working so they can't just bring me down any time they want. (Male, 64 years)

So I set up a ride with the VA Transport and that's a pain in the ass because if they got ten people in there, I can't go in there with my walker and my leg wrapped up. I tried it once and it was devastating. (Male, 57 years)

\section{Limited PCP involvement in the medical decision to return to the hospital}

Some participants considered the decision to seek urgent care without input from the $\mathrm{PCP}$ - the person ostensibly the most knowledgeable about and most trusted by the patient-was due to these logistical/ structural barriers or tendencies to ignore symptoms (especially for the loner and 'hardcore' patients) until there seemed to be no option but to go to the emergency department.

It happened on a weekend and I had no choice but to go to the Emergency Room... Well, like this last time. I started getting pains in my stomach and naturally, my daughter and my son said, "You better go". (Male, 82 years)

Well, like sometimes I let it go too long [and so go to the Emergency Room]. I don't get to where I'm supposed to be till, it's not too late but it's late. (Male, 60 years)

\section{Perceived inevitability of the rehospitalisation}

Perhaps the most surprising issue was that most patients believed that their readmission was inevitable because they had a chronic, degenerative or terminal illness. Only two participants believed that their readmission could have been prevented. One attributed his kidney failure to what he believed to be inadequate monitoring of his lab data, and the other was convinced that he had been discharged too soon.

I was following [the doctor's] orders to take MiraLax twice a day and still I wound up here. I don't get it. (Male, 82 years)

I don't think [the doctor] could've done anything... Because it was- my heart hadn't ever done anything like that before. So there's no way any doctor would have known. (Male, 76 years)

\section{Discharge-associated themes}

Several themes that may have contributed to participants' readmission concerned the discharge process and postdischarge services. These included beliefs about premature discharge, inadequate or poorly understood information about postdischarge plans or poorly coordinated postdischarge services.

\section{Perceived premature discharge}

Participants noted persistent symptoms or insufficient rehabilitation as contributing to their rehospitalisation. For some, their strongly expressed desire to return home may have played an important role in early discharge and their ultimate readmission. For example, it was unclear in the case of one participant whether he had shared with his providers that he continued to be symptomatic at the time of his first discharge. In another case resulting in readmission after falling at home, it was unclear whether the patient would have received training on using his walker had he not left the hospital against medical advice.

I come down with C. diff. They kept me in the hospital for approximately a week and a half. They sent me home, even though I still had C. diff. They never tested me and...I was at home. I had a problem with diarrhea and so on and so forth. (Male, 64 years)

My congestive heart failure was so bad that they started treating that. And treated that for about 9 weeks. And then sorta had fully recovered, but I hadn't. I was too weak. And I left the hospital 1 day, and when I got home, I realized I shouldn't be home, so I came back. (Male, 74 years)

Response to question about the readmission: I needed more (inpatient) physical therapy, rehab. (Male, 65years)

\section{Insufficient or poorly understood information about postdischarge} plans

Several participants thought that insufficient or unclear information at the time of discharge may have contributed to their readmission. It is possible that some (eg, passive/accepting or 'hardcore' patient types) may not have asked questions to clarify any confusion concerning the discharge plans.

I know exactly what I can eat, how to prepare my foods, and a little bit more than I did the very first time they released me. (Male, 64 years)

There's about 15 pills that I have to take and God knows what they are and for what they're used for, because nobody has explained what they are used for. (Male, 87 years)

\section{Inadequate postdischarge services}

Finally, some complained that their postdischarge treatment was either poorly coordinated, inadequate to address their postdischarge service needs or not covered by their insurance. 
[VA staff] told me, "You go home and take care of your treatment at home. Okay? When you get there, your nurse will be waiting for you. They'll come every day to do it for you. Put in the machine and get it ready". They lied. Nobody showed up. (Male, 64 years)

Yes, they set me up for a visiting nurse or visiting physical therapist but it lasted for about 3 days each, which didn't accomplish much. (Male, 69 years)

\section{Patient recommendations about how to reduce readmission risk}

Most participants were pleased with their VA healthcare but recommended several ways to further improve care and reduce risk of rehospitalisation. These included increasing involvement of PCPs, reducing transportation and distance barriers to primary and specialty care, expanding predischarge services and improving coordination of postdischarge services.

\section{Improve access to PCP and to expedite more urgent cases}

Most participants had great respect and trust for the primary care providers, and many suggestions concerned ways in which to reduce challenges to interacting with them both within and outside the hospital. These included requiring a call-back within 24 hours for urgent complaints or being able to 'rush the system' in cases where quicker scheduling of services or procedures are required.

I wish I could talk directly [to the primary care team], pick up the phone and you gotta go through like with the Veteran's Service...Everything goes to an answering machine. (Male, 57 years)

Instead of, maybe have two or three appointments or testings or something set up real close together to find out, to get a good grip on what's going on and then you know is it something that we need to really move with or is it something we can kind of put the brakes on and slow down a bit. (Male, 60 years)

I mean you've got a 48 hours I think system for notification or for where they got to return the information back and, if anything, I would like to see some way where there's a red button that you could hit that would be an emergency; in other words if I got a problem that needs to be addressed right away, is there something in the system, the computer or telephone where that can happen. (Male, 68 years)

And now [because of delays in scheduling the procedure], they're looking at removing the complete bile duct system, so they kind of went from a minor surgery to a very complex, major surgery. (Male, 60 years)

Reduce barriers associated with long distances or limited transportation services

Recommendations to reduce challenges to accessing primary and specialty outpatient services involved arranging for efficient and courteous transportation services and reducing travel distances for such services.

...the transportation with the VA is a very poor system when it comes to handicapped persons like myself. They have to come up with something better for us because we are handicapped. (Male, 57 years)

They never asked me, Do you need money for transportation?...Like at some places, they got the cashier downstairs and I see some guys get money for transportation. (Male, 82 years)

\section{Improve postdischarge service provision}

Participants believed that better coordination and expansion of predischarge and postdischarge services could reduce readmission rates. They thought that patients at high risk for readmission (eg, recent infections, serious disease) should have more aggressive follow-up and in-home care. They also suggested expanding inpatient nutrition and physical therapy services to better prepare them for returning home, expanding postdischarge services and improving the efficiency of the process for covering such services.

And what they should've done was...keep me in the hospital long enough to know or, what they should've done, they should've told me not to eat solid food. They should've told me I should be eating a pureed diet. (Male, 64 years)

In response to a question about whether visiting nurse services would have prevented one participant's readmission: I would have ended up in the same position [of being readmitted], but sooner. (Male, 65 years)

\section{DISCUSSION}

Current VA discharge procedures require providing patients with telephone numbers and instructions about reasons to call or return to the hospital. The VA has mandated that all patients be contacted by their primary care team within 48 hours of discharge, yet our major finding was that participants largely did not appear to interact with the medical system prior to deciding to return to the hospital. Rather, they remained at home until they or their family felt the person was 'sick enough' to warrant hospitalisation. This finding is consistent with other studies noting that individuals from disadvantaged $^{27}{ }^{31}$ or veteran ${ }^{13} 32$ groups often prefer to access healthcare through the emergency department or make triage decisions independently. Interventions to improve health literacy may help patients better understand discharge instructions and reasons to contact their health providers postdischarge. ${ }^{33}{ }^{34}$ However, the tendency of patients such as the loners or 'hardcore' identified in our study to delay contacting their provider may be so firmly entrenched that it may be difficult to change attitudes or behaviour. 
Although further research is needed to determine the generalisability of our findings, the data suggest that greater involvement of and interaction with the primary care team may help to reduce rehospitalisation rates. This can be addressed in several ways. First, to address gaps in postdischarge care, we suggest placing a second postdischarge follow-up call 5-7 days after the first for high-risk patients such as those with frailty-related diagnoses ${ }^{10}$ or complicated medication regimens. This could identify patients at risk of readmission earlier and connect them with needed outpatient services when appropriate. For patients who are receiving home-based care-be they in their own home or in a long-term assisted living facility, follow-up calls to the private or community-based organisation providing services may further decrease risk of readmission. Periodic calls during the first few weeks after discharge could verify patients' status and that they are receiving the services ordered in the discharge plan.

Our participants reported trusting their PCPs, valued receiving unified messages when there were multiple providers, and were disappointed that their PCP had virtually no input during their hospitalisation. Limited interaction with primary care was also reported among non-VA patients, ${ }^{35} 36$ where only $24 \%$ of geriatric, family or internal medicine physicians care for their patients both in and out of the hospital. ${ }^{37}$ Given the VA goal of creating patient-centred medical homes, ${ }^{38}$ we recommend initiatives to increase PCP involvement in both the inpatient and outpatient settings. Currently, no formal procedure or requirement exists for involving PCPs in their VA patients' inpatient care. Some VA systems have implemented interventions to improve transitions of care between the inpatient and outpatient settings. ${ }^{39}$ Although logistically complex, formal involvement of PCPs at admission and during hospitalisation may further reduce readmission risk and should be evaluated in further work.

The perceived logistical/structural challenges appeared to influence decisions about seeking emergency versus primary care services. Although VA data indicate that VACT exceeds benchmark standards in access to care for primary care urgent visits, ${ }^{40}$ interventions to expand access to primary care have shown mixed results in reducing readmissions. ${ }^{1041}$ Our data suggest the need for expanding such access. This could be accomplished by adding an on-call medical provider $24 / 7$ for patients to consult in cases needing timely action but not necessarily emergency services.

Similarly and consistent with another study, ${ }^{8}$ physical distance from the main VA medical centre appeared to be a barrier to seeking care. Since the time of our study, the VA has expanded local access to non-VA care.$^{42}$ However, given veterans' appreciation of VA care and desire for coordinated care, implementing specialty and ancillary services within the CBOCs might also be considered.

It was unclear whether perceptions about not receiving adequate services predischarge and postdischarge represented a lack rather than misunderstanding of discharge instructions. This issue should be studied further, possibly using ethnographic observation methods. We also recommend carefully assessing patients' discharge readiness, predischarge service needs (eg, nutrition education, physical therapy, polypharmacy), postdischarge living situation and support system, medication management strategies and need for assistance. This assessment process should begin at admission and continue through discharge when discharge instructions should be reviewed with the patient and persons who will assist with their care. As mentioned previously, it should also involve interactions with private or community-based service organisations when needed.

Several study limitations exist. Our interviews were conducted from September 2013 to October 2014. Since then, the VA system has addressed some of the barriers noted by our participants. These changes (which occurred at approximately the same time as this study) included reducing call centre response times, instituting weekend hours and PCP urgent visits and expanding access to specialty services with the Veterans Choice bill. It will be important to assess whether the perceived barriers noted in our study have been reduced with these interventions. Second, the study included mainly older and Caucasian participants from a single medical centre, and as such, the findings may not apply to other veteran populations. Future research is needed to determine whether additional readmission themes exist among women and minority populations or in other geographic regions. Third, we did not collect data from patients' medical records and were therefore unable to validate their reports of medical events; the self-reported data may have been subject to recall and social desirability bias. Finally, as in any qualitative study, interpretation of the study results, although grounded in the data, is viewed through the epistemological and ontological lenses of the researchers performing the analysis. Given the multidisciplinary nature of the research team, we believe that our perspectives and training were diverse and permitted a broader and more balanced consideration of the data.

This study identified perceived barriers that may contribute to rehospitalisation among a sample of older veteran patients such as limited contact with the medical system prior to returning to the hospital, limited access to care due to clinic hours and distance and the adequacy of services prior to and following discharge. The data further identified strategies that may reduce risk of rehospitalisation, including proactive postdischarge outreach to high-risk patients, patient education regarding the role of primary care, increased access to outpatient care locally and during non-business hours and improved discharge planning.

Acknowledgements The authors would like to thank the participants for providing their unique perspectives on possible factors contributing to their recent readmissions and to Drs Amish Desai, Alexandra Norcott and Alexander Pine, the internal medicine residents who served as additional interviewers on this project.

Contributors SMA provided substantial contribution to the research design and data collection and analysis. She led the manuscript development process, provided final approval of the document and agrees to be accountable for all aspects of the work. LEG led the data analysis and provided substantial contribution to 
manuscript preparation, research design and data collection and analysis. She provided final approval of the document and agrees to be accountable for the data analysis portion of the work. RSB provided substantial contribution to the research design of the study and provided final approval of the document. She agrees to be accountable for all aspects of the work.

Funding This study was funded by VHA Office of Academic Affiliations (OAA). Competing interests None declared.

Patient consent Obtained.

Ethics approval VA Connecticut Healthcare System West Haven IRB.

Provenance and peer review Not commissioned; externally peer reviewed.

Data sharing statement The interview guide and codebook used during data analysis are available to anyone on email request to the corresponding author.

Open access This is an open access article distributed in accordance with the Creative Commons Attribution Non Commercial (CC BY-NC 4.0) license, which permits others to distribute, remix, adapt, build upon this work non-commercially, and license their derivative works on different terms, provided the original work is properly cited and the use is non-commercial. See: http://creativecommons.org/ licenses/by-nc/4.0/

(C) Article author(s) (or their employer(s) unless otherwise stated in the text of the article) 2018. All rights reserved. No commercial use is permitted unless otherwise expressly granted.

\section{REFERENCES}

1. Benbassat J, Taragin M. Hospital readmissions as a measure of quality of health care: advantages and limitations. Arch Intern Med 2000;160:1074-81.

2. Carey K, Stefos T. The cost of hospital readmissions: evidence from the VA. Health Care Manag Sci 2016;19:241-8.

3. Nuti SV, Qin L, Rumsfeld JS, et al. Association of admission to veterans affairs hospitals vs non-veterans affairs hospitals with mortality and readmission rates among older men hospitalized with acute myocardial infarction, heart failure, or pneumonia. JAMA 2016;315:582-92.

4. Weeks WB, West AN, Wallace AE, et al. Comparing the characteristics, utilization, efficiency, and outcomes of VA and nonVA inpatient care provided to VA enrollees: a case study in New York. Med Care 2008;46:863-71.

5. Neupane B, Walter SD, Krueger P, et al. Predictors of inhospital mortality and re-hospitalization in older adults with communityacquired pneumonia: a prospective cohort study. BMC Geriatr 2010;10:22

6. Targher G, Dauriz M, Laroche C, et al. In-hospital and 1-year mortality associated with diabetes in patients with acute heart failure: results from the ESC-HFA heart failure long-term registry. Eur J Heart Fail 2017;19:54-65.

7. Farsi M, Ridder G. Estimating the out-of-hospital mortality rate using patient discharge data. Health Econ 2006;15:983-95.

8. Holloway JJ, Medendorp SV, Bromberg J. Risk factors for early readmission among veterans. Health Serv Res 1990;25:213-37.

9. Moore CD, Gao K, Shulan M. Racial, income, and marital status disparities in hospital readmissions within a veterans-integrated health care network. Eval Health Prof 2015;38:491-507.

10. Pugh JA, Wang CP, Espinoza SE, et al. Influence of frailty-related diagnoses, high-risk prescribing in elderly adults, and primary care use on readmissions in fewer than 30 days for veterans aged 65 and older. J Am Geriatr Soc 2014;62:291-8.

11. Tang VL, Halm EA, Fine MJ, et al. Predictors of rehospitalization after admission for pneumonia in the veterans affairs healthcare system. $J$ Hosp Med 2014:9:379-83.

12. Enguidanos S, Coulourides Kogan AM, Schreibeis-Baum H, et al. "Because i was sick": seriously ill veterans' perspectives on reason for 30-day readmissions. J Am Geriatr Soc 2015;63:537-42.

13. Stephens C, Sackett N, Pierce R, et al. Transitional care challenges of rehospitalized veterans: listening to patients and providers. Popul Health Manag 2013;16:326-31.

14. Greysen SR, Stijacic Cenzer I, Auerbach AD, et al. Functional impairment and hospital readmission in medicare seniors. JAMA Intern Med 2015;175:559-65.

15. Greysen SR, Harrison JD, Kripalani S, et al. Understanding patientcentred readmission factors: a multi-site, mixed-methods study. BMJ Qual Saf 2017;26:33-41.
16. Burke LG, Jha AK. Patients' functional status and hospital readmissions. JAMA Intern Med 2015;175:565-6.

17. Hoyer EH, Needham DM, Atanelov L, et al. Association of impaired functional status at hospital discharge and subsequent rehospitalization. J Hosp Med 2014;9:277-82.

18. Morandi A, Bellelli G, Vasilevskis EE, et al. Predictors of rehospitalization among elderly patients admitted to a rehabilitation hospital: the role of polypharmacy, functional status, and length of stay. J Am Med Dir Assoc 2013;14:761-7.

19. Dickinson $\mathrm{H}$, Carico $\mathrm{C}$, Nuño $\mathrm{M}$, et al. Unplanned readmissions and survival following brain tumor surgery. J Neurosurg 2015;122:61-8.

20. Holzwarth R, Huber D, Majkrzak A, et al. Outcome of gastric bypass patients. Obes Surg 2002;12:261-4.

21. Chaiyachati $\mathrm{KH}$, Gordon K, Long T, et al. Continuity in a VA patientcentered medical home reduces emergency department visits. PLoS One 2014;9:e96356.

22. Singh S, Lin YL, Kuo YF, et al. Variation in the risk of readmission among hospitals: the relative contribution of patient, hospital and inpatient provider characteristics. J Gen Intern Med 2014;29:572-8.

23. Green UR, Dearmon V, Taggart H. Improving transition of care for veterans after total joint replacement. Orthop Nurs 2015;34:79-86.

24. Kansagara D, Chiovaro JC, Kagen D, et al. Transitions of care from hospital to home: an overview of systematic reviews and recommendations for improving transitional care in the veterans health administration. Washington DC: Department of Veterans Affairs, 2015.

25. Paquin AM, Salow M, Rudolph JL. Pharmacist calls to older adults with cognitive difficulties after discharge in a tertiary veterans administration medical center: a quality improvement program. J Am Geriatr Soc 2015;63:571-7.

26. U.S. Department of Veterans Affairs. 2015 annual report. USA: VA New England Healthcare system: U.S. Government Printing Office, 2016.

27. Long T, Genao I, Horwitz LI. Reasons for readmission in an underserved high-risk population: a qualitative analysis of a series of inpatient interviews. BMJ Open 2013;3:e003212.

28. Braun V, Clarke V. Using thematic analysis in psychology. Qual Res Psychol 2006;3:77-101.

29. Braun V, Clarke V. Successful qualitative research: a practical guide for beginners. Thousand Oaks CA: SAGE Publications Ltd, 2013.

30. U.S. Department of Veterans Affairs. Celebrating women's history month. Secondary 664 Celebrating women's history month 2015. 2015 http://www.connecticut.va.gov/CONNECTICUT/features/ Women_Veterans_Health_Program.asp

31. Kangovi S, Barg FK, Carter T, et al. Understanding why patients of low socioeconomic status prefer hospitals over ambulatory care. Health Aff 2013;32:1196-203.

32. Stevenson CW, Pori D, Payne K, et al. Hearing the veteran's voice in congestive heart failure readmissions. Prof Case Manag 2015;20:177-85.

33. Maniaci MJ, Heckman MG, Dawson NL. Functional health literacy and understanding of medications at discharge. Mayo Clin Proc 2008:83:554-8.

34. Rosenow EC. Patients' understanding of and compliance with medications: the sixth vital sign? Mayo Clin Proc 2005;80:983-7.

35. Horwitz LI, Moriarty JP, Chen C, et al. Quality of discharge practices and patient understanding at an academic medical center. JAMA Intern Med 2013;173:1715-22.

36. McAlearney AS. Hospitalists and family physicians: understanding opportunities and risks. J Fam Pract 2004;53:473-81.

37. Jones KC, Whatley MM. Hospitalists: a growing part of the primary care workforce. Secondary hospitalists: a growing part of the primary care workforce. 2016 https://www.aamc.org/download/462728/data/ june2016hospitalistsagrowingpartoftheprimarycareworkforce.pdf

38. Rosland AM, Nelson $\mathrm{K}$, Sun $\mathrm{H}$, et al. The patient-centered medical home in the veterans health administration. Am J Manag Care 2013;19:e263-72.

39. Moy NY, Lee SJ, Chan T, et al. Development and sustainability of an inpatient-to-outpatient discharge handoff tool: a quality improvement project. Jt Comm J Qual Patient Saf 2014;40:219-27.

40. US Department of Veterans Affairs. Strategic analytics for improvement and learing (SAIL). Secondary Strategic analytics for improvement and learing (SAIL). 2016 https://www.va. gov/QUALITYOFCARE/measure-up/Strategic_Analytics_for_ Improvement and Learning SAIL.asp

41. Leppin AL, Gionfriddo MR, Kessler M, et al. Preventing 30-day hospital readmissions. A systematic review and meta-analysis of randomaized trials. JAMA Int Med 2014;174:1095-107.

42. HealthNet Federal Services. Secondary December 15, 2016. 2014 https://www.hnfs.com/content/hnfs/home/va/home/provider/ veterans-choice-program.html 